\title{
SH-SAWs in layered Piezoelectric Structures Carrying an Array of Microbeams
}

\author{
Lili Yuan \\ School of Architectural, Civil Engineering and \\ Environment, Ningbo University, 818 Fenghua Road \\ Ningbo, Zhejiang 315211, China \\ e-mail:yuanlili@nbu.edu.cn
}

\section{Xian Fu}

School of Mechanical Engineering and Mechanics, Ningbo University, 818 Fenghua Road, Ningbo, Zhejiang 315211, China

e-mail:fuyaozm@163.com

\begin{abstract}
Shear horizontal surface acoustic waves (SHSAWS) propagation in layered piezoelectric structure carrying an array of microbeams is studied in this paper. Based on the three-dimensional piezoelectricity theory, the coupling wave equations are obtained and solved by analytical method with consideration of both electrically open and short conditions. The beams are modeled using the Euler-Bernoulli theories when they are bending during the piezoelectric structures in shear motion. The effects of the microbeams on the phase velocity are presented and discussed in detail. From the results, we can find the phase velocity decrease with the non-dimensional wave number, while it is increase with the number of the microbeams. The geometric parameters of the cantilever beams, such as Young's modulus, diameter and length have evident effect on the phase velocity, we can find that the phase velocity decrease with length-diameter ratio of the cantilever beams. The method and results presented in the paper could be useful to the analysis and design of surface acoustic wave devices.
\end{abstract}

Keywords- SH-SAW; dispersion; piezoelectric; microbeam; layered structure

\section{INTRODUCTION}

In 1911, Love analyzed a layered structure consisting of an isotropic substrate covered with an isotropic thin film, solid-coupled at their interface, and the conclusion is drawn that shear horizontal surface waves (SH-SAWs) would propagate in the thin film and attenuate along thickness of the substrate if the velocity of the bulk shear wave in the thin film is less than that in the substrate [1]. These shear surface waves are now known as the Love waves and their polarization is perpendicular to the sagittal plane formed by both the normal to the interface of a medium and the wave vector in the direction of wave propagation. Another SH-SAWs is so-called B-G surface acoustic wave which was first discovered by Bleustein and Gulyaev simultaneously in 1968-1969 [2-3], which can exist only on certain cuts and propagate in certain directions of piezoelectric crystals. It is well known that piezoelectric materials are widely used in acoustic wave devices such as sensors, actuators, and transducers for their strong coupling between electric and mechanical constitutive behavior since White invented the interdigital

\author{
Benjie Ding \\ School of Mechanical Engineering and Mechanics, \\ Ningbo University, 818 Fenghua Road, Ningbo, \\ Zhejiang 315211, China \\ e-mail:dingbenjie@163.com
}

transducers utilized for transmitting and receiving surface acoustic waves in 1965 [5]. There are numerous investigations have been taken on SH-SAWs in piezoelectric media due to their extensive applications [67].

Recently, due to the extensive effort on micro- and nano-technologies, various micro- or nano-scale beam arrays can be made using different techniques [8-9]. These new structures have great potentials for new devices such as efficient microneedles, MEMS actuators and biosensors, and so on [10]. It is urgent and important to study the effects of microbeams on vibrations of structures or wave propagation in structures due to the design of resonators, sensors and actuators. Liu et al. [11] analyzed multiobjective design optimization of electrostatically actuated microbeam resonators with and without parameter uncertainty. Liu et al. [12] investigated shear vibration of a rotated Y-cut quartz crystal plate carrying an array of microbeams with their bottoms fixed to the top surface of the plate. The plate was modeled by the theory of anisotropic elasticity and the beams were modeled by the Euler-Bernoulli theory for beam bending, and a frequency equation that determines the resonant frequencies of the structure was derived. Zhang et al. [13] studied thicknessshear vibration of an elastic plate carrying an array of rigid microbeams with their bottoms attached to the top surface of the plate. The plate was modeled by the couple-stress theory of elasticity to properly take into account both the shear forces and the bending moments at the bottoms of the beams. However, in the above-mentioned researches, there is no paper about SH-SAW propagation in layered piezoelectric structures carrying an array of microbeams. It is necessary to analyze the surface acoustic waves in layered structures with consideration of an array of microbeams due to their potential applications.

In this paper, the propagation of SH-SAWs in layered piezoelectric structures carrying an array of microbeams is investigated. The microbeams are modeled by the EulerBernoulli theories when they are bending during the piezoelectric structures in shear motion. The dispersion relations are calculated and discussed in detail. 


\section{FORMULATION OF THE PROBLEM}

The layered piezoelectric structure carrying an array of microbeams is illustrated in Fig .1., where a half-space piezoelectric substrate is covered by a thin elastic layer. We here only consider the so-called anti-plane wave propagation problem.
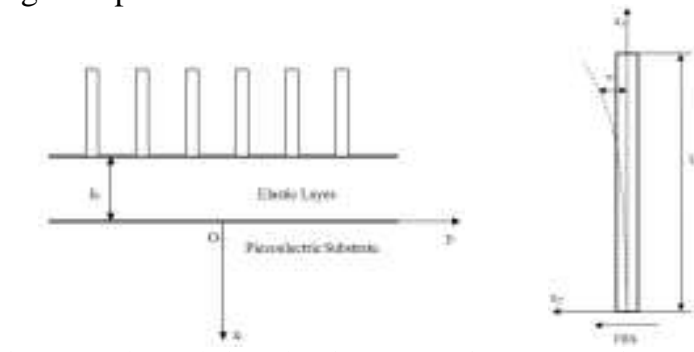

Figure 1. A layered piezoelectric structure carrying an array of microbeams distribution at the bottom of the beams can be neglected and we obtain [23]

$$
\begin{aligned}
& E I v^{\prime \prime \prime \prime}+\hat{\rho} S=0, \\
& \theta=v^{\prime}, V=E I v^{\prime \prime \prime}, M=E I v^{\prime \prime},
\end{aligned}
$$

where $\theta$ is the rotation or slope, $M$ is the bending moment, $v\left(x_{1}\right)$ is the deflection curve, and $V$ is the shear force. $E$ is the Young's modulus, $\hat{\rho}$ the mass density, and $I, S$ are the moment of inertia and area of the beam cross-section, respectively. We consider the time harmonic factor and can find in a straightforward manner that the solution to Equation (12) can satisfy $M(L)=0, V(L)=0$ and $\theta(0)=0$ is

$$
v=C f\left(x_{1}, E I, \hat{\rho} S, L, \omega\right) \exp [i k(y-c t)],
$$

where $\mathrm{C}$ is an undetermined constant and

$$
\begin{aligned}
& f=\cos \alpha\left(x_{1}-L\right)+\cosh \alpha\left(x_{1}-L\right)+\gamma\left[\sin \alpha\left(x_{1}-L\right)\right. \\
& \left.+\sinh \alpha\left(x_{1}-L\right)\right], \\
\alpha= & \left(\frac{\hat{\rho} S \omega^{2}}{E I}\right)^{1 / 4}, \gamma=\frac{\sinh \alpha L-\sin \alpha L}{\cos \alpha L+\cosh \alpha L} .
\end{aligned}
$$

From the above equations, we can get the shear force at the bottom as

$$
V(0)=C E I f^{\prime \prime \prime}(0, E I, \hat{\rho} S, L, \omega) \exp [i k(y-c t)],
$$

\section{BOUNDARY CONDITIONS}

The shear forces at the bottoms of many small beams effectively form a distributed shear stress on the layer surface and thereby affecting velocity or frequency of the waves. It was shown that through the beam arrayfrequency effect, information about the beam array can be extracted. Assume the number density of the beams per unit area of the elastic surface is N. The traction-free and electrically open conditions at the free surface can be given by

$$
\begin{aligned}
& w(-h)=-v(0), T_{x z}(-h)=-N V(0), \\
& \phi_{x}(-h)=\phi_{x}^{0}(-h), D_{x}(-h)=D_{x}^{0}(-h) .
\end{aligned}
$$

The traction-free and electrically shorted conditions at the free surface can be given by

$$
\begin{aligned}
& w(-h)=-v(0), T_{x z}(-h)=-N V(0), \\
& \phi(-h, y, t)=0 .
\end{aligned}
$$

The continuity conditions at the elastic layer and the piezoelectric substrate are written as

$$
\begin{array}{ll}
w\left(0^{-}\right)=w^{(\mathrm{m})}\left(0^{+}\right), & T_{x z}\left(0^{-}\right)=T_{x z}^{(\mathrm{m})}\left(0^{+}\right), \\
\phi\left(0^{-}\right)=\phi^{(\mathrm{m})}\left(0^{+}\right), & D_{x}\left(0^{-}\right)=D_{x}^{(\mathrm{m})}\left(0^{+}\right) .
\end{array}
$$

\section{SOLUTION OF THE PROBLEM}

\section{A. Solutions in the piezoelectric substrate}

We consider the following solution of (4)

$$
\begin{aligned}
& w^{(\mathrm{m})}(x, y, t)=A^{m} \mathrm{e}^{-k b^{(\mathrm{m})} x} \exp [i k(y-c t)], \\
& \psi^{(\mathrm{m})}(x, y, t)=B^{m} \mathrm{e}^{-k x} \exp [i k(y-c t)],
\end{aligned}
$$

where $k$ is the wave number, $c$ is the phase velocity, $b^{(\mathrm{m})}=\sqrt{1-\rho^{m} c^{2} / c_{44}^{m *}} . A^{m}$ and $B^{m}$ are unknown constants. So 


\section{B. Solutions in the elastic layer}

The solutions of the displacement and the electric potential in the elastic layer are given by

$$
\begin{aligned}
& w(x, y, t)=\left(A_{1} \operatorname{coskb} x+A_{2} \operatorname{sinkb} x\right) \exp [i k(y-c t)], \\
& \phi(x, y, t)=\left(B_{1} \mathrm{e}^{-k x}+B_{2} \mathrm{e}^{k x}\right) \exp [i k(y-c t)],
\end{aligned}
$$

where $b=\sqrt{\rho c^{2} / c_{44}-1}, A_{1}, A_{2}, B_{1}$ and $B_{2}$ are unknown constants.

\section{THE PHASE VELOCITY EQUATION}

Inserting Eqs. (12), (13) and their corresponding components of stress and displacement into boundary and continuity conditions, we can obtain the algebraic equations of the unknowns $C=\left[A_{1}, A_{2}, A_{3}, A_{4}, A_{5}, A_{6}, A_{1}^{m}, A_{2}^{m}, A_{3}^{m}, A_{4}^{m}, A_{5}^{m}, A_{6}^{m}, A_{1}^{e}, A_{2}^{e}, A_{3}^{e}\right]^{T}$ i.e.

$$
[\mathbf{A}][C]=0 .
$$

In order to obtain the nontrivial solutions of the above mentioned unknown constants, the determinant of the coefficient matrix of these linear algebraic equations must equal zero, i.e. $|A|=0$. So the dispersive relations of the electrically open and shorted case can be obtained.

\section{NUMERICAL RESULTS}

For the numerical calculations, the piezoelectric material is assumed to be $\mathrm{LiNbO}_{3}$, and the elastic material is $\mathrm{SiO}_{2}$. Unless otherwise specified, the thickness of elastic layer is assumed to be $h=0.1 \mathrm{~mm}$ and the beams are considered of $\mathrm{ZnO}$ with $\hat{\rho}=5600 \mathrm{~kg} / \mathrm{m}^{3}, \mathrm{~L}=1 \mu \mathrm{m}, \mathrm{N}=1 \times 10^{11} / \mathrm{m}^{2}, \quad \mathrm{E}=40 \mathrm{GPa}$, and diameter $D=0.1 \mu \mathrm{m}$. For the cantilever beams $\omega$ are given in [14]

$$
\omega=\beta_{m}^{2} \frac{D}{4} \sqrt{\frac{E I}{A \rho}}=\beta_{m}^{2} \frac{D}{4} \sqrt{\frac{E}{\rho}}, \quad m=1,2,3 \mathrm{~L}
$$

Here we only consider the first two resonances and unless otherwise specified, here we take the first frequency into account.

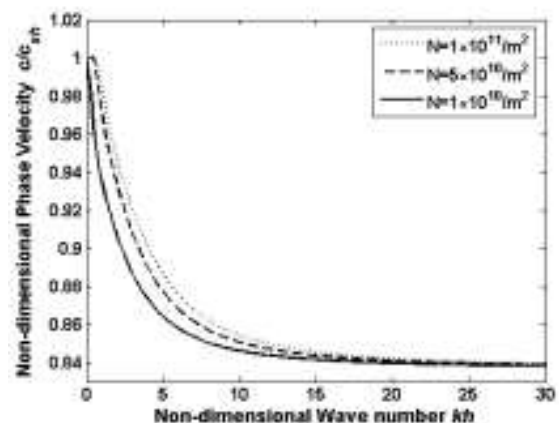

Figure.3 Dispersive relationship for shorted case with different $N$

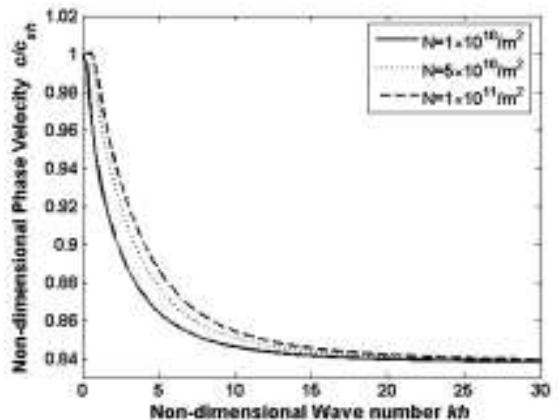

Figure.4 Dispersive relationship for open case with different $N$

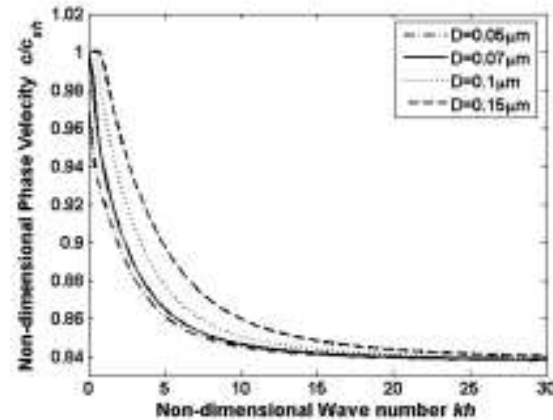

Figure.5 Dispersive relationship for shorted case with different $D$

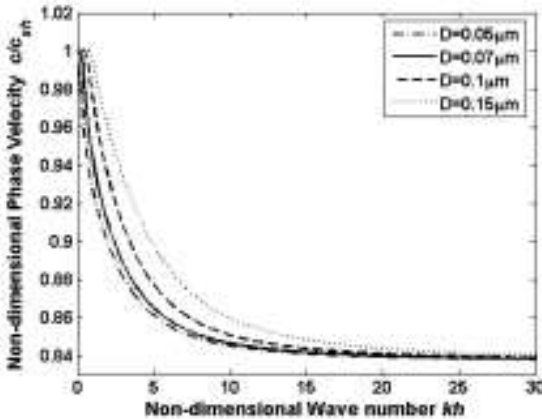

Figure.6 Dispersive relationship for open case with different $D$

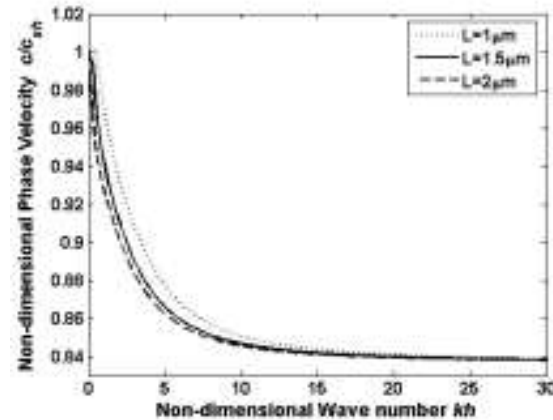

Figure.7 Dispersive relationship for shorted case with different $L$

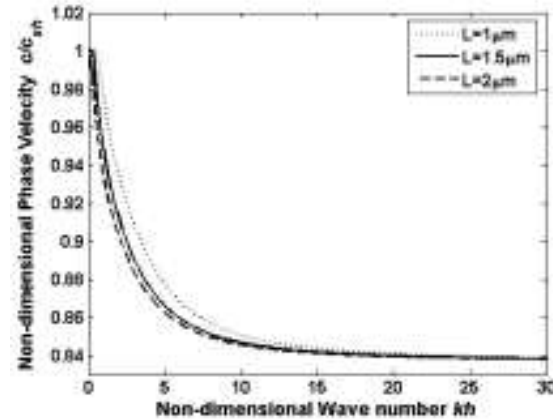

Figure.8 Dispersive relationship for open case with different $L$ 
Fig. 3 and Fig.4 show the phase velocity of the firstorder mode for electrically open and shorted cases with different $N$, respectively. The calculation results for different number of microbeams are figured for a contrast. We can find the phase velocity decrease with the nondimensional wave number, while it is increase with the number of the microbeams.

In order to show effect of geometric parameters of the cantilever beams, such as diameter and length, Fig.5, Fig.6, Fig. 7 and Fig. 8 show the phase velocity of the first-order mode for electrically open and shorted cases, respectively. we can find that they both have evident effect on the phase velocity. The phase velocity decrease with length-diameter ratio of the cantilever beams.

\section{CONCLUSIONS}

The properties of $\mathrm{SH}$ surface acoustic waves in perfectly bonded piezoelectric layered structures covered a microbeam array are studied in this paper. The solutions of dispersion relations are obtained with electrically open or shorted conditions by analytical method. The effects of the microbeams on the phase velocity are presented and discussed in detail. From the results we can find that the phase velocity increases distinct when the layered structure carrying an array of microbeams. Furthermore, we can find that the phase velocity increase with the geometric parameters of the microbeams. The analytical method and the results can be useful for the design of the ultrasonic brush and sensors.

\section{ACKNOWLEDGMENT}

This research are supported by a grant from the Mechanics Initiative, a COE Program of Zhejiang Provincial Government in the Department of Mechanics and Engineering Science, Ningbo University (Grant No. xklx1508), Research Project of Ningbo University (No. XKL14D2071) and Ningbo Natural Science Foundation (No. 2014A610171) .

\section{REFERENCES}

[1] A. E. H. Love, Some Problems of Geodynamics, Cambridge University Press, London, 1911.

[2] J. L. Bleustein, "A new surface wave in piezoelectric materials, " Appl. Phys. Lett., vol. 13, 1968, pp. 412-413.

[3] Y. V. Gulyaev, "Electroacoustic surface waves in solids, " JETP Lett., vol. 9, 1969, pp. 37-38.

[4] L. L. Yuan, J. K. Du, T. F. Ma, J. Wang, "Effects of Viscous Liquid on SH-SAW in Layered Magnetoelectric Structures, "Ultra, vol. 53, 2013, pp. 808-814.

[5] R. M. White, F. W. Voltmer, "Direct piezoelectric coupling to surface elastic waves, ” Appl Phys Lett, 1965, pp. 7-314.

[6] F.L. Guo, R. Sun, "Propagation of Bleustein-Gulyaev wave in $6 \mathrm{~mm}$ piezoelectric materials loaded with viscous liquid, " Int. J. Solids Struct, vol. 45(13), 2008 , pp. 3699-3710.

[7] S. S. Myung, J. K. Yeon, "The effect of initial stress on the propagation behavior of $\mathrm{SH}$ waves in piezoelectric coupled plates, "Ultra, vol. 51, 2011, pp. 489-495.

[8] G. Ulises, L. Yirong, J. E. Gregory, A.S. Henry, "Effect of $\mathrm{ZnO}$ nanowire morphology on the interfacial strength of nanowire coated carbon fibers, " Compos. Sci. Technol., vol. 71, 2011, pp. 946-954.

[9] M. Shuaishuai, L. Rong, L. Changpeng, X. Wei, X.L. Gou, "Facile synthesis of $\mathrm{ZnO}$ nanorod arrays and hierarchical nanostructures for photocatalysis and gas sensor applications, " J. Hazard. Mater., vol. 192,2011 , pp. 730-740.

[10] L. W. Zhong, "ZnO nanowire and nanobelt platform for nanotechnology, ” Mater. Sci. Eng., R 64, 2009, pp. 33-71.

[11] M. Liu, M. Kurt, M. F. Dan, "Multi-objective design optimization of electrostatically actuated microbeam resonators with and without parameter uncertainty, "Reliab eng syst safe 92, 2007, pp. 1333-1343.

[12] N. Liu, J. S. Yang, J. Wang, "Shear Vibration of a Crystal Plate Carrying an Array of Microbeams," Philos. Mag. Lett., vol. 91,2011, pp. 572-581.

[13] R. Y. Zhang, J. M. Xie, Y. T. Hu, J. S. Yang, X. D. Chen "Thickness-shear vibration of an elastic plate carrying an array of rigid microbeams with consideration of couple stresses, " Int. J. Engng. Sci., vol. 51,2012 , pp. 179-189.

[14] J. M. Gere, Mechanics of materials (5th ed.), Pacific Grove, CA: Brooks/Cole, 2001. 